\title{
In eigener Sache: Info DaF dankt Dr. Armin Wolff für jahrzehntelanges Engagement
}

Nach fast vier Jahrzehnten kontinuierlicher und engagierter Mitarbeit verabschiedet sich Schriftleiter Dr. Armin Wolff mit dieser Ausgabe von Info DaF. Er gehörte 1974, als die erste Ausgabe der »Informationen Deutsch als Fremdsprache (Info $D a F$ ) erschien, zu den wichtigsten Initiatoren dieser Fachzeitschrift und hat ihre Entwicklung sowie ihr Profil maßgeblich beeinflusst. Info DaF hat bis heute sehr von Armin Wolffs weitreichenden und vielfältigen Erfahrungen und Kenntnissen auf dem Gebiet des Faches Deutsch als Fremdsprache profitieren können. Sein Engagement reicht zurück bis an das Ende der 60er Jahre, als er das noch neue Lehrgebiet an der Universität Regensburg aufbaute und leitete; es ist u. a. geprägt durch seinen aktiven Einsatz im Vorstand des "Arbeitskreises Deutsch als Fremdsprache $\ll(\mathrm{AkDaF})$ und später im Vorstand sowie im Beirat des daraus hervorgegangenen »Fachverbandes Deutsch als Fremdsprache $(\mathrm{FaDaF}), \mathrm{zu}$ dessen Gründungsmitgliedern er zählte. Weit über die Grenzen der Hochschule engagierte er sich in der Diskussion um die Rolle von DaF an den Hochschulen. Dass Info DaF als Forum des lebendigen Austausches zwischen Theorie und internationaler Praxis heute einen festen Platz in der DaF-Welt hat, ist nicht zuletzt Armin Wolffs unermüdlichem Einsatz zu verdanken.

Die Redaktion spricht Armin Wolff ihren großen Dank aus und wünscht ihm vor allem Gesundheit und eine aktive Zeit ohne Redaktionsschlüsse und andere drängende Publikationstermine.

Ab der nächsten Ausgabe (1/2012) übernimmt Professor Dr. Christian Krekeler die Schriftleitung von Info DaF (krekeler@htwg-konstanz.de). Vertreten wird er von Professor Dr. Uwe Koreik. 